\title{
On certain subclasses of multivalently meromorphic close-to-convex maps
}

\author{
by K. S. Padmanabhan (Madras)
}

\begin{abstract}
Let $M_{p}$ denote the class of functions $f$ of the form $f(z)=1 / z^{p}+$ $\sum_{k=0}^{\infty} a_{k} z^{k}, p$ a positive integer, in the unit disk $E=\{|z|<1\}, f$ being regular in $0<|z|$ $<1$. Let $L_{n, p}(\alpha)=\left\{f: f \in M_{p}, \operatorname{Re}\left\{-\left(z^{p+1} / p\right)\left(D^{n} f\right)^{\prime}\right\}>\alpha\right\}, \alpha<1$, where $D^{n} f=$ $\left(z^{n+p} f(z)\right)^{(n)} /\left(z^{p} n !\right)$. Results on $L_{n, p}(\alpha)$ are derived by proving more general results on differential subordination. These results reduce, by putting $p=1$, to the recent results of Al-Amiri and Mocanu.
\end{abstract}

1. Introduction. Let $M_{p}$ denote the class of meromorphic functions $f$ in the unit disk $E=\{z:|z|<1\}$ having only a pole of order $p$ at $z=0$, of the form

$$
f(z)=\frac{1}{z^{p}}+\sum_{k=0}^{\infty} a_{k} z^{k}, \quad p \text { a positive integer. }
$$

We define

$$
D^{n} f(z)=\frac{1}{z^{p}(1-z)^{n+1}} * f(z)
$$

where $n$ is a non-negative integer and $*$ denotes Hadamard product. It can be verified that

$$
D^{n} f(z)=\frac{\left(z^{n+p} f(z)\right)^{(n)}}{z^{p} n !},
$$

where $f^{(n)}$ denotes the $n$th derivative of $f$ in the usual sense. Let $L_{n, p}(\alpha)$, $\alpha<1$, denote the class of functions $f \in M_{p}$ such that

$$
\operatorname{Re}\left\{\frac{-z^{p+1}}{p}\left(D^{n} f\right)^{\prime}\right\}>\alpha .
$$

1991 Mathematics Subject Classification: Primary 30C45.

Key words and phrases: meromorphic multivalently close-to-convex, differential subordination, convolution. 
Let

$$
I_{c}(f)(z)=\frac{c-p+1}{z^{c+1}} \int_{0}^{z} t^{c} f(t) d t, \quad \operatorname{Re} c>0 .
$$

For $p=1$, H. Al-Amiri and P. T. Mocanu [1] have shown that $L_{n+1,1}(\alpha) \subset$ $L_{n, 1}(r), r>\alpha$; and $I_{c}\left[L_{n, 1}(\alpha)\right] \subset L_{n, 1}(\delta), \delta>\alpha$. Furthermore, they proved that if $f \in M_{1}$ and $\operatorname{Re} c>0$ then

$$
\operatorname{Re}\left[-z^{2}\left(D^{n} f(z)\right)^{\prime}\right]>\alpha-(1-\alpha) \operatorname{Re} \frac{1}{c} \Rightarrow I_{c}(f) \in L_{n, 1}(\alpha) .
$$

Indeed they have proved certain more general results on differential subordination from which the above results are deduced.

In this paper, we obtain analogous results for $L_{n, p}(\alpha)$ when $p$ is any positive integer and the results obtained by Al-Amiri and Mocanu [1] can be deduced from our results when we put $p=1$. We also establish general results on differential subordination from which the results of Al-Amiri and Mocanu are deducible.

\section{Preliminary definitions and lemmas}

DeFinition 1. If $f$ and $g$ are analytic in $E$ and $g$ is univalent in $E$, then $f$ is said to be subordinate to $g$, written $f \prec g$, if $f(0)=g(0)$ and $f(E) \subset g(E)$.

Lemma A [3, 4]. Let $p(z)=p(0)+p_{n} z^{n}+\ldots$ be analytic in $E$ and $q$ analytic and univalent in $E$. If $p$ is not subordinate to the analytic function $q$ in $E$, then there exist points $z_{0} \in E$ and $\zeta_{0} \in \partial E$ such that (i) $p\left(z_{0}\right)=q\left(\zeta_{0}\right)$, (ii) $z_{0} p^{\prime}\left(z_{0}\right)=m \zeta_{0} q^{\prime}\left(\zeta_{0}\right)$, where $m \geq n$.

Lemma B $[3,4]$. Let the function $H: \mathbb{C}^{2} \rightarrow \mathbb{C}$ satisfy $\operatorname{Re} H(i s, t) \leq 0$ for real $s$ and $t \leq-n\left(1+s^{2}\right) / 2$, where $n$ is a positive integer. If $p(z)=$ $1+p_{n} z^{n}+\ldots$ is analytic in $E$ and $\operatorname{Re} H\left(p(z), z p^{\prime}(z)\right)>0$ for $z \in E$, then $\operatorname{Re} p(z)>0$ in $E$.

Definition 2. Let $z \in E, t \geq 0$. A function $L(z, t)$ is called a subordination chain if $L(\cdot, t)$ is analytic and univalent on $E$ for all $t \geq 0, L(z, \cdot)$ is continuously differentiable on $[0, \infty)$ for each $z \in E$, and $L(z, s) \prec L(z, l)$ for $0 \leq s<l$.

Lemma $\mathrm{C}[6]$. The function $L(z, t)=a_{1}(t) z+\ldots$, with $a_{1}(t) \neq 0$ and $\lim _{t \rightarrow \infty}\left|a_{1}(t)\right|=\infty$, is a subordination chain if and only if

$$
\operatorname{Re}\left[z \frac{\partial L}{\partial z} / \frac{\partial L}{\partial t}\right]>0 \quad \text { for } z \in E \text { and } t \geq 0 .
$$


Lemma D [7]. Let $\alpha, \beta \in \mathbb{R}, \alpha>0, g$ starlike univalent in $E$, and $h$ analytic in $E$ with $h(0)=1$ and $\operatorname{Re} h>0$ in $E$. Define

$$
B(\alpha, \beta)=\left\{F: F(z)=\left(\int_{0}^{z} h(t) g^{\alpha}(t) t^{i \beta-1} d t\right)^{1 /(\alpha+i \beta)}, z \in E\right\} .
$$

Then $G \in B(\alpha, \beta)$, where

$$
G(z)=\left[z^{-c} \int_{0}^{z} t^{c-1} F^{\alpha+i \beta}(t) d t\right]^{1 /(\alpha+i \beta)}, \quad c \in \mathbb{C}, \operatorname{Re} c>0 .
$$

REMARK. $F \in B(\alpha, \beta)$ is univalent and analytic in $E$ and is called a Bazilevič function. The class $B(1,0)$ consists of close-to-convex functions in $E$.

Definition 3. Let $H\left(p(z), z p^{\prime}(z)\right) \prec h(z)$ be a first order differential subordination. Then a univalent function $q$ is called its dominant if $p \prec q$ for all analytic functions $p$ that satisfy the differential subordination. A dominant $\bar{q}$ is called the best dominant if $\bar{q} \prec q$ for all dominants $q$. For the general theory of differential subordination and its applications we refer to $[5]$.

Lemma 1. Let $q$ be a convex univalent function in $E$ and $\operatorname{Re} c>0$. Let

$$
h(z)=q(z)+\frac{p+1}{c} z q^{\prime}(z)
$$

where $p$ is a positive integer. If $p(z)=1+a_{p+1} z^{p+1}+\ldots$ is analytic in $E$ and

$$
p(z)+\frac{1}{c} z p^{\prime}(z) \prec h(z),
$$

then $p(z) \prec q(z)$ and $q$ is the best dominant.

Proof. We can assume that $q$ is analytic and convex on $\bar{E}$ without any loss of generality, because otherwise we replace $q(z)$ by $q_{r}(z)=q(r z)$, $0<r<1$. These functions satisfy the conditions of the lemma on $\bar{E}$. We can prove that $p_{r}(z) \prec q_{r}(z)$, which enables us to obtain $p \prec q$ on letting $r \rightarrow 1$. Consider

$$
L(z, t)=q(z)+\frac{p+1+t}{c} z q^{\prime}(z), \quad z \in E, t \geq 0 .
$$

Then

$$
\frac{\partial L}{\partial t}=\frac{z q^{\prime}(z)}{c}, \quad \frac{\partial L}{\partial z}=q^{\prime}(z)+\frac{p+1+t}{c} z q^{\prime \prime}(z)+\frac{p+1+t}{c} q^{\prime}(z) .
$$

We have

$$
\operatorname{Re}\left(\frac{z \partial L / \partial z}{\partial L / \partial t}\right)=\operatorname{Re}\left\{c+(p+1+t)\left(1+z q^{\prime \prime}(z) / q^{\prime}(z)\right)\right\}>0,
$$


since $q$ is convex and $\operatorname{Re} c>0$. Hence $L(z, t)$ is a subordination chain by Lemma C. We have $L(z, 0)=h(z) \prec L(z, t)$ for $t>0$ and $L(\zeta, t) \notin h(E)$ for $|\zeta|=1$ and $t \geq 0$. If $p$ is not subordinate to $q$, then by Lemma A, there exist points $z_{0} \in E, \zeta_{0} \in \partial E$ and $m \geq p+1$ such that $p\left(z_{0}\right)=q\left(\zeta_{0}\right)$, $z_{0} p^{\prime}\left(z_{0}\right)=m \zeta_{0} q^{\prime}\left(\zeta_{0}\right)$ and so

$$
p\left(z_{0}\right)+\frac{1}{c} z_{0} p^{\prime}\left(z_{0}\right)=q\left(\zeta_{0}\right)+\frac{m}{c} \zeta_{0} q^{\prime}\left(\zeta_{0}\right)=L\left(\zeta_{0}, m-p-1\right) \notin h(E),
$$

which contradicts our assumption that $p(z)+\frac{1}{c} z p^{\prime}(z) \prec h(z)$. So we conclude that $p \prec q$. Consider $p(z)=q\left(z^{p+1}\right)$ to see that $q$ is the best dominant.

LEMMA 2. Let

$$
w=\frac{(p+1)^{2}+|c|^{2}-\left|(p+1)^{2}-c^{2}\right|}{4(p+1) \operatorname{Re} c}, \quad \operatorname{Re} c>0 .
$$

If $h$ is analytic in $E$ with $h(0)=1$ and

$$
\operatorname{Re}\left\{1+\frac{z h^{\prime \prime}(z)}{h^{\prime}(z)}\right\}>-w,
$$

and if $p(z)=1+a_{p+1} z^{p+1}+\ldots$ is analytic in $E$ and satisfies

$$
p(z)+\frac{1}{c} z p^{\prime}(z) \prec h(z),
$$

then $p(z) \prec q(z)$, where $q(z)$ is the solution of

$$
q(z)+\frac{p+1}{c} z q^{\prime}(z)=h(t), \quad q(0)=1,
$$

given by

$$
q(z)=\frac{c}{(p+1) z^{c /(p+1)}} \int_{0}^{z} t^{c /(p+1)-1} h(t) d t .
$$

Also $q$ is the best dominant of (5).

Proof. Using Lemma 1, we see that it is sufficient to show that $q$ is convex. First we note that $w \leq 1 / 2$. To see this we observe that $\operatorname{Re} c>0$ implies $|c-(p+1)|<|c+(p+1)|$. Multiplying by $|c-(p+1)|$ and simplifying, we get

$$
(p+1)^{2}+|c|^{2}-\left|(p+1)^{2}-c^{2}\right|<2 \operatorname{Re} c \cdot(p+1)
$$

whence $w \leq 1 / 2$.

If $c=p+1$, then $w=1 / 2$, and (4) implies that $h$ is close-to-convex and, by Lemma D, (7) implies that $q$ is also close-to-convex. So $q^{\prime}(z) \neq 0$ for $z \in E$ and the function

$$
P(z)=1+\frac{z q^{\prime \prime}(z)}{q^{\prime}(z)}=1+P_{1} z+P_{2} z^{2}+\ldots
$$


is analytic in $E$, with $P(0)=1$. From (6), on differentiation, we get

$$
(p+1) P(z)+c=c h^{\prime}(z) / q^{\prime}(z) .
$$

Again logarithmic differentiation and substitution for $z q^{\prime \prime}(z) / q^{\prime}(z)$ in terms of $P(z)$ yields

$$
P(z)+z P^{\prime}(z) /\left(P(z)+\frac{c}{p+1}\right)=1+\frac{z h^{\prime \prime}(z)}{h^{\prime}(z)} .
$$

Now let

$$
H(u, v)=u+\frac{v}{u+\frac{c}{p+1}}+w
$$

Then

From (8), (9) and (4) we obtain

$$
\begin{aligned}
\operatorname{Re} H(i s, t) & =\operatorname{Re}\left\{i s+\frac{t}{i s+\frac{c}{p+1}}+w\right\} \\
& =\operatorname{Re}\left\{\frac{t(p+1)(\bar{c}-(p+1) i s)}{|c+(p+1) i s|^{2}}+w\right\} \\
& =\frac{(p+1) t \operatorname{Re} c}{|c+(p+1) i s|^{2}}+w .
\end{aligned}
$$

$$
\operatorname{Re} H\left(P(z), z P^{\prime}(z)\right)>0, \quad z \in E .
$$

We proceed to show that $\operatorname{Re} H(i s, t) \leq 0$ for all real $s$ and $t \leq-\left(1+s^{2}\right) / 2$ :

(11) $\quad \operatorname{Re} H(i s, t)=\frac{(p+1) t \operatorname{Re} c}{|c+(p+1) i s|^{2}}+w$

$$
\begin{aligned}
\leq & -\frac{1}{|c+(p+1) i s|^{2}}\left\{s^{2}\left(\frac{p+1}{2} \operatorname{Re} c-(p+1)^{2} w\right)\right. \\
& \left.-2 s(p+1) w \cdot \operatorname{Im} c+\operatorname{Re} c \cdot \frac{p+1}{2}-w|c|^{2}\right\} .
\end{aligned}
$$

For $w$ given by (3), the coefficient of $s^{2}$ of the quadratic expression in $s$ in braces is positive. To check this, put $c=c_{1}+i c_{2}$ so that $\operatorname{Re} c=c_{1}, \operatorname{Im} c=c_{2}$. We have to verify that

$$
c_{1}>2(p+1) w=\frac{(p+1)^{2}+|c|^{2}-\left|(p+1)^{2}-c^{2}\right|}{2 c_{1}} .
$$

This inequality will hold if

$$
2 c_{1}^{2}+\left|(p+1)^{2}-c^{2}\right|>(p+1)^{2}+|c|=(p+1)^{2}+c_{1}^{2}+c_{2}^{2},
$$

that is, if

$$
\left|(p+1)^{2}-c^{2}\right|>(p+1)^{2}-\operatorname{Re} c^{2},
$$

which is obviously true. Further, the quadratic expression in $s$ is a perfect square for the assumed value of $w$. So from (11) we see that Re $H(i s, t) \leq 0$. 
Lemma B enables us to conclude from (10) that $\operatorname{Re} P(z)>0, z \in E$, that is,

$$
\operatorname{Re}\left\{1+\frac{z q^{\prime \prime}(z)}{q^{\prime}(z)}\right\}>0, \quad z \in E .
$$

So $q$ is convex and the proof is complete.

REMARK. If $c>0$, then $w=\frac{c}{2(p+1)}$ for $0<c \leq p+1$, and $w=\frac{p+1}{2 c}$ for $c>p+1$.

\section{Theorems and their proofs} let

Theorem 1. Let $q$ be a convex analytic function in $E$ with $q(0)=1$ and

$$
h(z)=q(z)+\frac{(p+1) z q^{\prime}(z)}{n+1}, \quad n \text { a positive integer. }
$$

If $f \in M_{p}$ and

$$
D^{n} f(z)=\frac{1}{z^{p}(1-z)^{n+1}} * f(z),
$$

then

$$
-\frac{z^{p+1}}{p}\left(D^{n+1} f\right)^{\prime} \prec h \Rightarrow-\frac{z^{p+1}}{p}\left(D^{n} f\right)^{\prime} \prec q
$$

and the latter subordination is best possible.

Proof. One can verify without difficulty the relation

$$
(n+1) D^{n+1} f=z\left(D^{n} f\right)^{\prime}+(n+p+1) D^{n} f .
$$

Set $P(z)=-\frac{z^{p+1}}{p}\left(D^{n} f\right)^{\prime}$. Differentiation gives

$$
p z P^{\prime}(z)=p(p+1) P(z)-z^{p+2}\left(D^{n} f\right)^{\prime \prime} .
$$

Differentiating (12) we obtain

$$
(n+1)\left(D^{n+1} f\right)^{\prime}=(n+p+2)\left(D^{n} f\right)^{\prime}+z\left(D^{n} f\right)^{\prime \prime} .
$$

Multiplying (14) by $-z^{p+1}$ and using (13) gives

$$
\frac{z P^{\prime}}{n+1}+P(z)=-\frac{z^{p+1}}{p}\left(D^{n+1} f\right)^{\prime} \prec h(z) .
$$

Moreover, $P(0)=1$ and $P^{\prime}(0)=0$. Indeed, $P(z)=1+P_{p+1} z^{p+1}+$ $P_{p+2} z^{p+2}+\ldots$ By Lemma 1 , we conclude that $P(z) \prec q(z)$ and $q$ is the best dominant.

THEOREM 2. Let $h$ be analytic in $E$ with

$$
h(0)=1, \quad \operatorname{Re}\left(1+\frac{z h^{\prime \prime}(z)}{h^{\prime}(z)}\right)>-w,
$$


where

$$
w= \begin{cases}(n+1) /(2(p+1)), & n=0,1, \ldots, p-1, \\ (p+1) /(2(n+1)), & n \geq p,\end{cases}
$$

$n$ being a positive integer. If $f \in M_{p}$, then

$$
-\frac{z^{p+1}}{p}\left(D^{n+1} f\right)^{\prime} \prec h \Rightarrow-\frac{z^{p+1}}{p}\left(D^{n} f\right)^{\prime} \prec q,
$$

where $q$ is the solution of

$$
q(z)+(p+1) \frac{z q^{\prime}(z)}{n+1}=h(z), \quad q(0)=1 .
$$

In fact, $q$ is given by

$$
q(z)=\frac{n+1}{(p+1) z^{(n+1) /(p+1)}} \int_{0}^{z} t^{(n+1) /(p+1)-1} h(t) d t
$$

and it is the best dominant.

Proof. The proof is immediate from Lemma 2 , with $c=n+1$, if we note that the value of $w$ for positive $c$ is given by the remark following the proof of Lemma 2.

Corollary 1. $L_{n+1, p}(\alpha) \subset L_{n, p}(r)$ for $\alpha<1$, where the best possible value of $r$ is given by

$$
r=r(\alpha, n)=2 \alpha-1+\frac{2(1-\alpha)}{p+1}(n+1) \int_{0}^{1} \frac{t^{(n-p) /(p+1)}}{1+t} d t>\alpha .
$$

Proof. Choose

$$
h(z)=\frac{1+z(2 \alpha-1)}{1+z}
$$

in Theorem 2. Then $h$ is convex and

$$
q(z)=\frac{n+1}{(p+1) z^{(n+1) /(p+1)}} \int_{0}^{z} t^{(n-p) /(p+1)} \frac{1+(2 \alpha-1) t}{1+t} d t .
$$

Since $\operatorname{Re} h(z)>\alpha$, the theorem asserts that

$$
\operatorname{Re}\left\{-\frac{z^{p+1}}{p}\left(D^{n+1} f\right)^{\prime}\right\}>\alpha \Rightarrow-\frac{z^{p+1}}{p}\left(D^{n} f\right)^{\prime} \prec q(z) .
$$

But $q$ is convex as observed in Lemma 2, has real coefficients in its Taylor expansion and is real for real $z$. Hence $q(E)$ is symmetric with respect to 
the real and thus $\operatorname{Re} q(z)>q(1)$ for $z \in E$. Moreover,

$$
\begin{aligned}
q(1) & =\frac{n+1}{p+1} \int_{0}^{1} t^{(n-p) /(p+1)} \frac{1+(2 \alpha-1) t}{1+t} d t \\
& =2 \alpha-1+\frac{2(1-\alpha)}{p+1}(n+1) \int_{0}^{1} \frac{t^{(n-p) /(p+1)}}{1+t} d t \\
& =r, \quad \text { say. }
\end{aligned}
$$

Evidently

$$
r>2 \alpha-1+\frac{2(1-\alpha)}{p+1}(n+1) \int_{0}^{1} \frac{t^{(n-p) /(p+1)}}{2} d t=\alpha .
$$

So, if $f \in L_{n+1, p}(\alpha)$ then

$$
\operatorname{Re}\left\{-\frac{z^{(p+1)}}{p}\left(D^{n+1} f\right)^{\prime}\right\}>\min _{|z|<1} \operatorname{Re} q(z)=q(1)=r,
$$

which means $f \in L_{n, p}(r)$.

REMARK. For $n=0$,

$$
r=2 \alpha-1+\frac{2(1-\alpha)}{p+1} \int_{0}^{1} \frac{t^{1 /(p+1)-1}}{1+t} d t .
$$

Denoting the integral by $I\left(\frac{1}{p+1}\right)$, we have $r=0$ if

$$
2 \alpha-1+\frac{2(1-\alpha)}{p+1} I\left(\frac{1}{p+1}\right)=0,
$$

that is, if

$$
2 \alpha\left(1-\frac{I(1 /(p+1))}{p+1}\right)=1-\frac{2}{p+1} I\left(\frac{1}{p+1}\right) .
$$

Denoting this value of $\alpha$ by $\alpha_{0}$, we find $L_{m, p}\left(\alpha_{0}\right) \subset L_{0, p}(0), m>0$. Now $L_{0, p}(0)$ consists of the functions $f$ for which

$$
\operatorname{Re}\left\{-\frac{z^{p+1}}{p} f^{\prime}\right\}>0,
$$

since $D^{0} f=z^{-p}(1-z)^{-1} * f(z)=f(z)$. So $L_{0, p}(0)$ is a subclass of the class of multivalently close-to-convex meromorphic functions in the unit disk introduced by A. E. Livingston [2], the associated meromorphically starlike function being $-1 / z^{p}$.

Theorem 3. Let $h$ be defined on $E$ by

$$
h(z)=q(z)+\frac{p+1}{c-p+1} z q^{\prime}(z),
$$


where $q$ is convex univalent in $E, h(0)=1$, and $c$ is a complex number with $\operatorname{Re} c>p-1$. If $f \in M_{p}$ and $F=I_{c}(f)$, where

$$
I_{c}(f)(z)=\frac{c-p+1}{z^{c+1}} \int_{0}^{z} t^{c} f(t) d t
$$

then

$$
-\frac{z^{p+1}}{p}\left(D^{n} f(z)\right)^{\prime} \prec h(z) \Rightarrow-\frac{z^{p+1}}{p}\left(D^{n} F(z)\right)^{\prime} \prec q(z)
$$

and the subordination is sharp.

Proof. From (17) we get

$$
(c+1) F(z)+z F^{\prime}(z)=(c-p+1) f(z) .
$$

If we use the facts $D^{n}\left(z F^{\prime}\right)=z\left(D^{n} F\right)^{\prime}$ and

$$
z\left(D^{n} F\right)^{\prime}=(n+1) D^{n+1} F-(n+p+1) D^{n} F,
$$

then (18) yields

$$
(c+1) D^{n} F+(n+1) D^{n+1} F-(n+p+1) D^{n} F=(c-p+1) D^{n} f
$$

or

$$
(c-n-p) D^{n} F+(n+1) D^{n+1} F=(c-p+1) D^{n} f .
$$

Set

so that

$$
P(z)=-\frac{z^{p+1}}{p}\left(D^{n} F\right)^{\prime}
$$

$$
p P^{\prime}(z)=-(p+1) z^{p}\left(D^{n} F\right)^{\prime}-z^{p+1}\left(D^{n} F\right)^{\prime \prime} .
$$

Differentiating (19) and using (21) we obtain

$$
p z P^{\prime}(z)+p(n+1) P(z)=-(n+1) z^{p+1}\left(D^{n+1} F\right)^{\prime} .
$$

Differentiating (20) and using (21), we can rewrite (22) in the form

$$
\frac{z p^{\prime}(z)}{c-p+1}+P(z)=-\frac{z^{p+1}}{p}\left(D^{n} f\right)^{\prime} \prec h(z) .
$$

Since $P(z)=1+P_{1} z^{p+1}+\ldots$, application of Lemma 1 shows that (23) implies $P(z) \prec q(z)$ and $q$ is the best dominant.

\section{THEOREM 4. Let}

$$
w=\frac{(p+1)^{2}+\left|c^{\prime}\right|^{2}-\left|(p+1)^{2}-c^{\prime 2}\right|}{4(p+1) \operatorname{Re} c^{\prime}}, \quad \operatorname{Re} c^{\prime}>0, c^{\prime}=c-p+1 .
$$

Let $h$ be analytic in $E$ and satisfy

$$
h(0)=1, \quad \operatorname{Re}\left\{1+\frac{z h^{\prime \prime}(z)}{h^{\prime}(z)}\right\}>-w .
$$


If $f \in M_{p}$ and $F=I_{c}(f)$ is defined by (17), then

$$
-\frac{z^{p+1}}{p}\left(D^{n} f(z)\right)^{\prime} \prec h(z) \Rightarrow-\frac{z^{p+1}}{p}\left(D^{n} F(z)\right)^{\prime} \prec q(z),
$$

where $q$ is the solution of the differential equation

$$
q(z)+\frac{p+1}{c-p+1} z q^{\prime}(z)=h(z), \quad q(0)=1,
$$

given by

$$
q(z)=\frac{c-p+1}{(p+1) z^{(c-p+1) /(p+1)}} \int_{0}^{z} t^{(c-p+1) /(p+1)-1} h(t) d t .
$$

Moreover, $q$ is the best dominant. find

Proof. Setting $P(z)=-\frac{z^{p+1}}{p}\left(D^{n} F\right)^{\prime}$ as in the proof of Theorem 3, we

$$
\frac{z P^{\prime}(z)}{c-p+1}+P(z)=-\frac{z^{p+1}}{p}\left(D^{n} F\right)^{\prime} \prec h(z) .
$$

An application of Lemma 2 with $c$ replaced by $c^{\prime}=c-p+1$ gives $P(z) \prec q(z)$, where $q$ is given by (24). The proof is complete.

Corollary. If $\alpha<1, \operatorname{Re} c>p-1$, and $I_{c}$ is defined by (17), then

$$
I_{c}\left(L_{n, p}(\alpha)\right) \subset L_{n, p}(\delta),
$$

where

$$
\delta=\min _{|z|=1} \operatorname{Re} q(z)=\delta(\alpha, c)
$$

and

$$
q(z)=\frac{c-p+1}{(p+1) z^{(c-p+1) /(p+1)}} \int_{0}^{z} t^{(c-p+1) /(p+1)}\left\{\frac{1+(2 \alpha-1) t}{1+t}\right\} d t,
$$

and the result is sharp. Also if $c$ is real and $c>p-1$, then

(26) $\delta(\alpha, c)=q(1)=2 \alpha-1+\frac{2(1-\alpha)}{p+1}(c-p+1) \int_{0}^{1} \frac{t^{(c-p+1) /(p+1)-1}}{1+t} d t$.

Proof. If we choose

$$
h(z)=\frac{1+z(2 \alpha-1)}{1+z}
$$

in the theorem, then $h$ is convex and we deduce from the theorem that

$$
\operatorname{Re}\left\{-\frac{z^{p+1}}{p}\left(D^{n} f(z)\right)^{\prime}\right\}>\alpha \Rightarrow-\frac{z^{p+1}}{p}\left(D^{n} F(z)\right)^{\prime} \prec q(z),
$$


where $q$ is given by (25), and so $I_{c}\left(L_{n, p}(\alpha)\right) \subset L_{n, p}(\delta)$. If $c$ is real and $c>p-1$, then observing that $q(E)$ is convex and symmetric with respect to the real axis, we get $\operatorname{Re} q(z)>\delta=q(1)$ given by $(26)$.

REMARK. If we take $c=(3 p-1) / 2$, then the integral in (26) reduces to

$$
\int_{0}^{1} \frac{t^{-1 / 2}}{1+t} d t=\frac{\pi}{2} .
$$

We have $\delta=2 \alpha-1+(1-\alpha) \pi / 2$, and $\delta=0$ if $\alpha=-(\pi-2) /(4-\pi)$. If

$$
\operatorname{Re}\left\{-\frac{z^{p+1}}{p}\left(D^{n} f\right)^{\prime}\right\}>-\frac{\pi-2}{4-\pi}
$$

then

$$
\operatorname{Re}\left\{-\frac{z^{p+1}}{p}\left(D^{n} F\right)^{\prime}\right\}>0,
$$

where

$$
F(z)=\frac{p+1}{2} \cdot \frac{1}{z^{(3 p+1) / 2}} \int_{0}^{z} t^{(3 p-1) / 2} f(t) d t .
$$

Theorem 5. Let $f \in M_{p}$ and let $I_{c}(f)$ be defined by (17). Let $\alpha<1$. If

$$
\operatorname{Re}\left\{-\frac{z^{p+1}}{p}\left(D^{n} f\right)^{\prime}\right\}>\alpha-(1-\alpha) \operatorname{Re} \frac{1}{c-p+1}
$$

then $I_{c}(f) \in L_{n, p}(\alpha)$.

Proof. Denote $I_{c}(f)$ by $F$ and put

$$
\frac{-z^{p+1}\left(D^{n} F(z)\right)^{\prime}}{p}=(1-\alpha) P(z)+\alpha .
$$

Using (20) and (12) we obtain after differentiation and simplification

$$
(c+2)\left(D^{n} F\right)^{\prime}+z\left(D^{n} F\right)^{\prime \prime}=(c-p+1)\left(D^{n} f\right)^{\prime} .
$$

Multiplying both sides of (28) by $z^{p+1}$ and using (27) we obtain

$$
\begin{aligned}
-\{(1-\alpha) P(z)+\alpha\} p(c+2)+p(p+1)\{(1-\alpha) & P(z)+\alpha\} \\
& -(1-\alpha) p z P^{\prime}(z) \\
= & (c-p+1) z^{p+1}\left(D^{n} f\right)^{\prime},
\end{aligned}
$$


or

$$
-\frac{z^{p+1}}{p}\left(D^{n} f\right)^{\prime}=(1-\alpha) P(z)+\alpha+(1-\alpha) \frac{z P^{\prime}(z)}{c-p+1} .
$$

So the inequality in the assumptions of the theorem becomes

$$
\operatorname{Re}\left\{(1-\alpha) P(z)+\frac{1-\alpha}{c-p+1}\left(z P^{\prime}(z)+1\right)\right\}>0, \quad z \in E .
$$

Since $P(z)=1+P_{p+1} z^{p+1}+\ldots$, in order to show that (30) implies that $\operatorname{Re} P(z)>0$ in $E$, it suffices to prove the inequality

$$
\operatorname{Re}\left\{(1-\alpha) i s+\frac{1-\alpha}{c-p+1}(t+1)\right\} \leq 0
$$

for all real $s$ and

$$
t \leq-\left(1+s^{2}\right) \frac{p+1}{2} \leq-\left(1+s^{2}\right),
$$

by Lemma B. Since $\operatorname{Re}(c-p+1)>0$, the inequality holds and so $\operatorname{Re} P(z)$ $>0$. In other words,

$$
\operatorname{Re}\left\{-\frac{z^{p+1}}{p}\left(D^{n} F\right)^{\prime}\right\}=\operatorname{Re}(1-\alpha) P(z)+\alpha>\alpha,
$$

or $F \in L_{n, p}(\alpha)$. The proof is complete.

REMARK. If $\alpha=0$, we conclude that

$$
\operatorname{Re}\left\{-\frac{z^{p+1}}{p}\left(D^{n} f\right)^{\prime}\right\}>-\operatorname{Re} \frac{1}{c-p+1} \Rightarrow \operatorname{Re}\left\{-\frac{z^{p+1}}{p}\left(D^{n} F\right)^{\prime}\right\}>0 .
$$

If moreover $n=0$ and $c=p$, we obtain the result: For $f \in M_{p}$,

$$
\operatorname{Re}\left\{-\frac{z^{p+1}}{p} f^{\prime}(z)\right\}>-1 \Rightarrow \operatorname{Re}\left\{-\frac{z^{p+1}}{p} F^{\prime}(z)\right\}>0,
$$

where $F(z)=z^{-p-1} \int_{0}^{z} t^{p} f(t) d t$.

\section{References}

[1] H. Al-Amiri and P. T. Mocanu, On certain subclasses of meromorphic close-toconvex functions, Bull. Math. Soc. Sci. Math. Roumanie 38 (86) (1994), 3-15.

[2] A. E. Livingston, Meromorphic multivalent close-to-convex functions, Trans. Amer. Math. Soc. 119 (1965), 167-177.

[3] S. S. Miller and P. T. Mocanu, Second order differential inequalities in the complex plane, J. Math. Anal. Appl. 65 (1978), 289-305.

[4] - - - Differential subordinations and univalent functions, Michigan Math. J. 28 (1981), 167-171.

[5] - - - The theory and applications of second order differential subordinations, Studia Univ. Babeş-Bolyai Math. 34 (1989), 3-33. 
[6] C. Pommerenke, Univalent functions, Vandenhoeck and Ruprecht, Göttingen, 1975.

[7] S. Ruscheweyh, Eine Invarianzeigenschaft der Basilevič-Funktionen, Math. Z. 134 (1973), 215-219.

M $73 / 2,31^{\text {st }}$ Cross Street

Besant nagar

Madras 600 090, India

E-mail: amlav@giasmdo1.vsnl.net.in

Reçu par la Rédaction le 9.10.1997 